Revue de droit comparé du travail et de la sécurité sociale

3 | 2018

La participation des travailleurs dans la grande entreprise privée et publique

\title{
La participation des travailleurs dans les grandes entreprises du secteur public en France
}

Sylvain Niquège

\section{CpenEdition}

\section{Journals}

Édition électronique

URL : https://journals.openedition.org/rdctss/1906

DOI : $10.4000 /$ rdctss. 1906

ISSN : 2262-9815

Éditeur

Centre de droit comparé du travail et de la sécurité sociale

Édition imprimée

Date de publication : 1 novembre 2018

Pagination : 108-117

ISSN : 2117-4350

Référence électronique

Sylvain Niquège, "La participation des travailleurs dans les grandes entreprises du secteur public en France », Revue de droit comparé du travail et de la sécurité sociale [En ligne], 3 | 2018, mis en ligne le 01 novembre 2021, consulté le 13 novembre 2021. URL : http://journals.openedition.org/rdctss/1906 DOI : https://doi.org/10.4000/rdctss. 1906

\section{(c) (i) (9)}

Revue de droit comparé du travail et de la sécurité sociale est mise à disposition selon les termes de la Licence Creative Commons Attribution - Pas d'Utilisation Commerciale - Pas de Modification 4.0 International. 


\title{
LA PARTICIPATION DES TRAVAILLEURS DANS LES GRANDES ENTREPRISES DU SECTEUR PUBLIC EN FRANCE
}

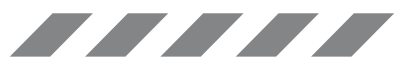

\begin{abstract}
There are undeniable singularities in the modes of participation of workers in large public-sector companies. However, these vary greatly from one public sector company to another, to the extent that we cannot assume the existence of a specific participation model that can be contrasted with that of private sector companies.
\end{abstract}

KEY WORDS : Participation, Public Company, Public Sector, Company with a Specific Employment Status, Participation Model.

\section{RÉSUMÉ}

Les modalités de la participation des travailleurs au sein des grandes entreprises du secteur public présentent d'incontestables singularités. Celles-ci varient cependant grandement d'une entreprise du secteur public à l'autre, si bien que l'on ne saurait conclure à l'existence d'un modèle spécifique de participation susceptible d'être opposé à celui que connaissent les entreprises du secteur privé.

MOTS CLÉS : Participation, entreprise publique, secteur public, entreprise à statut, modèle de participation. 
e thème de "la participation des travailleurs dans les grandes entreprises du secteur public » interroge l'existence éventuelle d'une pluralité de modèles de participation au sein du monde de l'entreprise ${ }^{1}$. Comme tout sujet, celui-ci suppose de s'entendre sur les termes de son énoncé.

Si elle présente parfois d'insondables difficultés, la définition de certains d'entre eux, déjà abordée dans le cadre d'autres interventions, ne donnera lieu qu'à de brèves remarques.

La participation, d'abord. On se contentera de rappeler qu'il s'agit en France d'un principe constitutionnel, garanti par l'alinéa 8 du Préambule de la Constitution de 1946, selon lequel «Tout travailleur participe, par l'intermédiaire de ses délégués, à la détermination collective des conditions de travail ainsi qu'à la gestion des entreprises». La participation renvoie donc à la fois au droit à la négociation collective et au droit des travailleurs de participer à la gestion des entreprises. Néanmoins, ce droit (il n'est pas le seul) n'a finalement guère que le sens que le législateur veut bien lui donner, dès lors que l'article 34 de la Constitution charge ce dernier de déterminer "les conditions et les garanties» de la mise en œuvre de l'alinéa 8 du Préambule ${ }^{2}$. Si l'on veut bien se dégager quelque peu de ce support constitutionnel, la participation peut être présentée sous deux angles complémentaires. L'un, matériel, interroge ce à quoi le travailleur a vocation à participer. Son champ s'élargit alors. II inclut toujours la négociation des conditions de travail, la discussion des choix stratégiques et le contrôle de l'entreprise, mais encore la participation aux résultats de celle-ci. L'autre, institutionnel, se rapporte aux modalités de la participation, c'est-à-dire aux moyens de la représentation et aux procédures de participation, en lien avec d'autres principes constitutionnels et, en particulier, la liberté syndicale ${ }^{3}$. Les lignes qui suivent seront consacrées à ces deux dimensions.

L'entreprise, ensuite. II n'y a rien de bien original à rappeler que sa définition varie en fonction du prisme juridique par lequel on l'aborde (droit commercial, droit du travail, etc.). On s'en tiendra donc à la double référence régulièrement faite, d'une part, à l'aspect organisationnel (un regroupement autonome de moyens faisant « ensemble » - locaux, matériels, capitaux, personnels), d'autre part, à la finalité poursuivie, ou plutôt à la nature

1 Cette question fait donc l'impasse sur celle, sans doute mieux balisée, de la distinction entre la participation dans la fonction publique et la participation en dehors de la fonction publique. Cette distinction n'est cependant pas étrangère, on le verra, à la problématique ici traitée.

2 Voir notamment $\mathrm{O}$. Dutheillet de Lamothe, "Les normes constitutionnelles en matière sociale ", Cahiers du Conseil constitutionnel, n² 29 - octobre 2010.

3 Alinéa 6 du PC 1946 : «Tout homme peut défendre ses droits et ses intérêts par l'action syndicale et adhérer au syndicat de son choix». 
de l'activité menée, à savoir une activité économique, elle-même définie par référence à

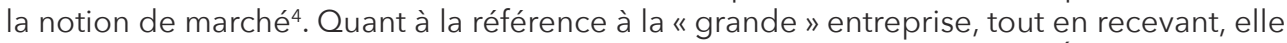
encore, diverses déclinaisons selon la branche du droit concernée (aides d'État, commande publique, plan de sauvegarde de l'emploi...), elle implique généralement une combinaison de seuils tenant au nombre de travailleurs et au chiffre d'affaires réalisé ${ }^{5}$. L'on assumera une part d'arbitraire en évoquant uniquement les entreprises qui nourrissent un lien avec l'État, à savoir les entreprises dites "nationales ", par opposition aux entreprises «locales " qui n'impliquent que les collectivités territoriales et/ou leurs établissements.

Ce lien avec l'État est central car il constitue la principale porte d'entrée vers la notion de secteur public, sur laquelle repose la singularité du sujet ici traité et dont la littérature juridique n'a de cesse, faute de définition légale rigoureuse, de souligner l'indétermination ${ }^{6}$. En effet, et de façon peut-être contre-intuitive, le secteur public renvoie moins à un ensemble identifié de marchés qu'à la part prépondérante que peuvent prendre les collectivités publiques au sein d'entreprises diverses, quel que soit le segment économique sur lequel elles sont positionnées. Cette approche organique de la notion étant posée, il est encore possible d'en affiner les contours en s'accordant sur une série d'indices.

Le premier est négatif et tient à l'affirmation selon laquelle le secteur public ne renvoie pas à une forme juridique d'entreprise en particulier. Aussi peut-on y inclure, outre les établissements publics, diverses formes de sociétés commerciales de droit privé, telles les sociétés d'économie mixte ${ }^{7}$. Ce faisant, l'expression de "secteur public » déborde le champ de la loi n 83-675 du 26 juillet 1983 relative à la démocratisation du secteur public, loi dont les dispositions portent d'abord sur les établissements publics ayant une activité industrielle et commerciale. On gardera néanmoins à l'esprit qu'avant qu'une ordonnance ne soit consacrée à la gouvernance et aux opérations sur le capital des sociétés à participation publique ${ }^{8}$, ces dernières se trouvaient incluses dans le champ de la loi de 1983. Aussi, il serait bien hasardeux d'inférer de l'absence d'évolution de l'intitulé de la loi de 1983 l'intention du législateur de réduire l'expression de "secteur public » aux seuls établissements publics?.

4 Ce qui correspond peu ou prou à la définition de l'entité économique par la directive 2001/23/CE du Conseil du 12 mars 2001 («ensemble organisé de moyens, en vue de la poursuite d'une activité économique, que celle-ci soit essentielle ou accessoire »).

5 À titre d'exemple, voir la recommandation de la Commission du 6 mai 2003 concernant la définition des micro, petites et moyennes entreprises.

6 Entre autres exemples, voir D. Linotte, R. Romi, Droit public économique, 7 e éd., 2012, Lexinexis, p. 337 ( "La définition juridique de ce que l'on entend par entreprise publique n'est pas vraiment figée »). Il en va de même pour la définition voisine de la notion d'entreprise publique. Sur les difficultés que pose celle-ci dans le domaine de la commande publique, voir L. Rapp, "Qu'est-ce qu'une 'entreprise publique' ? Questions sur l'article 11-2 de l'ordonnance du 23 juillet 2015 », AJDA 2017.1993.

7 La diversité des formes juridiques des entreprises du secteur public est plus importante encore au niveau local (sociétés publiques locales, sociétés d'économie mixte à opération unique, etc.).

8 Ordonnance $n^{\circ} 2014-948$ du 20 août 2014 relative à la gouvernance et aux opérations sur le capital des sociétés à participation publique.

9 C'est encore cette portée, large, que revêt l'utilisation de l'expression de « secteur public » dans les intitulés du Code du travail, que les dispositions qui s'ensuivent renvoient à la loi de 1983 (article L. 2144-1) ou concernent notamment « les entreprises publiques et les établissements publics à caractère industriel ou commercial » (article L. 2233-1). 
Le second indice, plus positif, tend à préciser la teneur et les modalités du contrôle que doit exercer la personne publique (l'État, en l'occurrence) sur une entreprise pour que celle-ci soit considérée comme relevant du secteur public. Traditionnellement, et hors les établissements publics, le droit interne privilégie le critère de la possession directe ou indirecte de la majorité du capital de l'entreprise considérée par une ou plusieurs personnes publiques ${ }^{10}$. D'autres approches, plus réalistes, tiennent compte de l'influence dont bénéficient une ou plusieurs personnes publiques sur les procédures et les choix de l'entreprise (vote au conseil d'administration, désignation des membres de l'organe d'administration / direction / surveillance, approbation, contrôle économique et financier...). On en trouve trace dans le droit de l'Union européenne mais aussi en droit interne ${ }^{11}$.

Quoi qu'il en soit des mérites de telle ou telle de ces approches du secteur public, elles conduisent toutes (et c'est là le principal dans le cadre de cette étude), à scruter une fois encore, en cette occasion au sein du champ économique, la frontière entre le public et le privé et ses effets éventuels sur le droit applicable. Précisément, la question est de savoir s'il y a ou non de réelles différences, du point de vue de la participation des travailleurs, entre les entreprises du secteur public et celles du secteur privé voire, le cas échéant, un «mieux disant participatif ». Deux séries de réflexions, que l'on veut complémentaires, seront menées. La première se rapporte à la nature de l'entreprise du secteur public. Elle conduit à se demander si, en tant que telle, celle-ci a une incidence identifiable et homogène sur les modalités de la participation. La seconde conduit, au sein même de l'entreprise du secteur public, à observer l'incidence que peut avoir sur les modalités de la participation la nature juridique de la relation de travail qui lie les travailleurs à leurs employeurs.

\section{I - NATURE DE L'ENTREPRISE DU SECTEUR PUBLIC ET PARTICIPATION DES TRAVAILLEURS}

À la question de savoir si la nature d'entreprise du secteur public a une incidence significative sur les modalités de la participation des travailleurs, précisément s'il y a un modèle juridique de participation propre aux grandes entreprises du secteur public, la réponse apparaît assez claire. C'est non.

Cela supposerait en effet l'application d'un corpus homogène de règles singulières considérées comme indissociables de la présence dominante de l'État, ce qui n'est pas le cas. Il suffira de rappeler que l'application d'un ensemble de règles distinctes du droit commun, se rattachant le cas échéant à ce que l'on appelle le droit public, n'a de sens que s'agissant d'activités, de finalités et/ou de méthodes elles-mêmes singulières. II y a bien

10 En ce sens, voir Conseil d'État, L'action économique des personnes publiques, étude annuelle 2015, La documentation française, p 147. De même, voir J.-M. Sauvé, Introduction aux Entretiens du Conseil d'État en droit public économique, 10 juin 2016,

http://www.conseil-etat.fr/content/download/64729/584861/version/1/file/20160610 Les\%20 entreprises\%20publiques\%20SRE.pdf. Le vice-président du Conseil d'État souligne qu' u une entreprise, qui n'est pas une personne publique, appartient au secteur public, si la majorité au moins de son capital est détenue, directement ou indirectement, par des personnes publiques. »

11 Art. L133-1 CJF, relatif au « contrôle des entreprises publiques » : " La Cour des comptes contrôle les établissements publics de l'Etat à caractère industriel et commercial. / Elle contrôle les sociétés dans lesquelles l'Etat détient la majorité du capital social ou des voix dans les organes délibérants ou sur lesquelles il exerce, directement ou indirectement, un pouvoir prépondérant de décision ou de gestion.» 
longtemps que le juge administratif a posé le principe selon lequel lorsqu'une personne publique agit « dans les mêmes conditions qu'un industriel ordinaire » (qu'une personne privée en général), elle doit être soumise au droit privé, et les litiges qui naissent de ladite activité relever de la compétence du juge judiciaire ${ }^{12}$. De sorte qu'elle soit de droit public ou, a fortiori, de droit privé, l'entreprise du secteur public nourrit avec ses agents des relations de droit privé régies par les dispositions du Code du travail ${ }^{13}$.

Ceci trouve naturellement à s'appliquer en matière de participation, même si le législateur a pris soin de le préciser expressément pour les établissements publics, dont la personnalité publique pouvait faire naître le doute. L'article L. 2211-1 du Code du travail, relatif au champ de la négociation collective et aux conventions et accords collectifs de travail, prévoit ainsi que « Les dispositions du présent livre sont applicables aux employeurs de droit privé [donc aux sociétés commerciales, sans exclusive] ainsi qu'à leurs salariés. / Elles sont également applicables: $1^{\circ}$ Aux établissements publics à caractère industriel et commercial; $2^{\circ}$ Aux établissements publics à caractère administratif lorsquiils emploient du personnel dans les conditions du droit privé ». L'article L. 2311-1 du Code du travail, relatif au champ d'application des dispositions relatives au comité social et économique, en fait de même. L'article L. 2111-1 du Code du travail, qui délimite celui des dispositions relatives aux syndicats professionnels, obéit à la même logique, quoique dans des termes quelque peu différents ${ }^{14}$. Quant à la participation aux résultats de l'entreprise, la démarche est énumérative puisqu' « un décret en Conseil d'Etat détermine les établissements publics de l,État à caractère industriel et commercial et les sociétés, groupements ou personnes morales, quel que soit leur statut juridique, dont plus de la moitié du capital est détenue directement par l'État, qui sont soumis aux dispositions du présent titre » (article L. 3321-1 du Code du travail) ${ }^{15}$.

Cette application des principales dispositions du Code du travail relatives à la participation des travailleurs aux entreprises du secteur public ne doit cependant pas conduire à éluder le fait que la présence de la puissance publique est rarement neutre, et qu'il est rare qu'elle ne produise aucun effet.

Tout d'abord, le Code du travail consacre certaines dispositions spécifiques aux entreprises relevant de la loi du 26 juillet 1983 relative à la démocratisation du secteur public, c'est-à-dire les établissements publics de l'État. C'est le cas en matière de négociation relative à l'exercice du droit syndical ${ }^{16}$. Ensuite et surtout, il ménage des possibilités d'adaptation, en particulier pour les établissements publics. L'article L. 2311-1 précise que les dispositions relatives au comité social et économique (comme avant lui les dispositions se rapportant au délégué du personnel et au comité d'entreprise), "peuvent, compte tenu

12 TC, 22 janvier 1921, Société commerciale de l'Ouest africain, Les grands arrêts de la jurisprudence administrative, $19^{\circ}$ éd. Dalloz, 2013, p. 223.

13 II en va de même des relations de l'entreprise du secteur public avec ses clients, qui sont des relations de droit privé régies par les règles du droit privé, telles que celles qui figurent dans le code de la consommation (à propos de l'interdiction des clauses abusives, voir CE, 11 juillet 2001, Société des eaux du Nord, $n^{\circ}$ 221458).

14 "Les dispositions du présent livre sont applicables aux employeurs de droit privé ainsi qu'à leurs salariés. / Elles sont également applicables au personnel des personnes publiques employé dans les conditions du droit privé $»$.

15 Voir le décret n87-948 du 26 novembre 1987.

16 Article L. 2144-1 et L. 2144-2. 
des caractères particuliers de certains des établissements mentionnés aux $1^{\circ}$ et $2^{\circ}$ [les établissements publics] et des instances de représentation du personnel éventuellement existantes, faire l'objet d'adaptations, par décrets en Conseil d'Etat, sous réserve d'assurer les mêmes garanties aux salariés de ces établissements ». Cette précision fait notamment écho aux particularismes des "entreprises à statut ", que l'on peut brièvement présenter comme des entreprises dont les agents sont des agents de droit privé mais qui relèvent, dans le même temps, d'un statut négocié prenant la forme de textes propres à l'entreprise et susceptible de déroger, dans les domaines prévus par la loi, aux dispositions du code du travail17. L'article L. 2111-1 alinéa 2 du Code du travail précise d'ailleurs que les dispositions relatives aux syndicats professionnels « sont également applicables au personnel des personnes publiques employé dans les conditions du droit privé, sous réserve des dispositions particulières ayant le même objet résultant du statut qui régit ce personnel ». Un bon exemple en est donné par la SNCF, I'article L. 2102-2 du Code des transports prévoyant que «La SNCF, SNCF Réseau et SNCF Mobilités emploient des salariés régis par un statut particulier élaboré dans des conditions fixées par décret en Conseil d'Etat. / La SNCF, SNCF Réseau et SNCF Mobilités peuvent également employer des salariés sous le régime des conventions collectives ». Un certain nombre de dérogations ou d'adaptations au code du travail sont ainsi annoncées à l'article L. 2101-4 du même Code, aux termes duquel "Le livre III de la deuxième partie du Code du travail relatif aux institutions représentatives du personnel s'applique au groupe public ferroviaire constitué de la SNCF, de SNCF Réseau et de SNCF Mobilités nonobstant toute disposition contraire du statut particulier mentionné à l'article L. 2101-2, sous réserve des dispositions spécifiques prévues à la présente section ${ }^{18}$. Quant aux conventions collectives, dont l'article L. 2233-1 du Code du travail prévoit qu'elles peuvent déterminer les conditions d'emploi et de travail ainsi que les garanties sociales des personnels des entreprises publiques, elles ne sont pas exclues par un éventuel statut, mais sont susceptibles de le compléter ou d'en préciser les termes dans les limites fixées par lui (article L. 2233-2 du Code du travail) ${ }^{19}$.

Ces dérogations et adaptations au droit commun de la participation ne sont pas l'apanage des établissements publics. Ce sont ainsi l'ensemble des entreprises du secteur public qui présentent certaines particularités en matière de participation des travailleurs aux organes de direction et de contrôle (conseil d'administration et conseil de surveillance), particularités qui trouvent leur origine dans le principe du «tripartisme " posé à la Libération. Concernant les établissements publics industriels et commerciaux, l'article 5 de la loi $n^{\circ} 83-675$ du 26 juillet 1983 relative à la démocratisation du secteur public précise que « Le conseil d'administration ou de surveillance comprend: / $1^{\circ}$ des représentants de l'Etat nommés par décret; / $2^{\circ}$ des personnalités choisies (...) / $3^{\circ}$ des représentants élus

17 CE, 11 oct. 2010, n³27660, DA n³, mars 2011, comm. 31, note T. Lahalle.

18 S'ensuivent une série de dérogations (articles L. 2101-5 et L. 2101-6) aux règles de droit commun relatives au comité social et économique central d'entreprise ou à la désignation des délégués syndicaux centraux.

19 Rappelant ce point pour les personnels de la SNCF, article L. 2101-3 du Code des transports : "Par dérogation aux articles L. 2233-1 et L. 2233-3 du Code du travail, pour les personnels de la SNCF, de SNCF Réseau et de SNCF Mobilités régis par un statut particulier, une convention de branche ou un accord professionnel ou interprofessionnel ayant fait l'objet d'un arrêté d'extension ou d'élargissement peut compléter les dispositions statutaires ou en déterminer les modalités d'application, dans les limites fixées par le statut particulier. » De même, pour les personnels des entreprises électriques et gazières, article L. 161-1 du Code de l'énergie. 
des salariés ». Le nombre de ces représentants des salariés, qui « ont les mêmes droits et obligations que les autres membres du conseil d'administration ou de surveillance » (article 21), doit «être égal au moins au tiers du nombre des membres du conseil dradministration ou du conseil de surveillance ${ }^{20}$. Concernant les sociétés anonymes, c'est l'article 7 de l'ordonnance $n^{\circ} 2014-948$ du 20 août 2014, relative à la gouvernance et aux opérations sur le capital des sociétés à participation publique, qui prévoit, sous certaines conditions, que dans les sociétés dont l'État détient directement plus de la moitié du capital « le conseil d'administration, le conseil de surveillance ou l'organe délibérant en tenant lieu comprend un tiers de représentants des salariés $»^{21}$.

Difficile néanmoins de déduire de ces spécificités une singularité de nature des entreprises du secteur public en matière de participation. Si les grandes entreprises du secteur public ne peuvent guère être associées à un " modèle » de participation, c'est encore parce qu'en leur sein, la nature juridique changeante de la relation de travail qui lie le travailleur à son employeur conduit à son tour à introduire de la diversité dans les dispositifs de participation.

\section{II - NATURE DE LA RELATION DE TRAVAIL ET PARTICIPATION DES TRAVAILLEURS}

Outre les considérations qui précèdent, la construction d'un modèle juridique de participation propre aux grandes entreprises du secteur public se heurte à l'absence d'homogénéité de leur personnel.

Cette diversité juridique des personnels pourrait surprendre dès lors que, dans la logique matérielle déjà évoquée, les personnels des entreprises publiques sont, ou devraient être des agents de droit privé. Bien sûr, ce principe a toujours connu des exceptions. On sait ainsi que dans un établissement public industriel et commercial, le directeur des services, comme le chef de la comptabilité quand il a la qualité de comptable public, ont la qualité d'agent public ${ }^{22}$. Reste que ces exceptions ne prêtent pas à conséquence sur le terrain de la participation. II en va différemment de ce que d'aucuns appellent le phénomène $d^{\prime}$ ' hybridation des personnels », propre à certaines grandes entreprises du secteur public où se côtoient agents de droit privé, agents publics et fonctionnaires ${ }^{23}$.

20 Par opposition, dans le secteur privé, voir les articles L. 225-27 s. du Code de commerce.

21 L'article 10 de la loi n $90-568$ du 2 juillet 1990 relative à l'organisation du service public de La Poste et à France Télécom rappelle ainsi que "L'ordonnance n 2014-948 du 20 août 2014 relative à la gouvernance et aux opérations sur le capital des sociétés à participation publique s'applique à La Poste ", avant de préciser que "Dès lors qu'une personne morale de droit public, autre que l'Etat, visée au I de l'article 1er-2 de la présente loi détient une part du capital de La Poste, le conseil d'administration de La Poste est composé (...) pour un tiers, de représentants des salariés élus dans les conditions prévues au chapitre II du titre II de la loi n 83-675 du 26 juillet 1983 ».

22 CE Sect., 8 mars 1957, Jalencques de Labeau, Leb. p. 158.

23 Le secteur marchand n'a pas le monopole de cette "hybridation", qui concerne tout autant, sinon plus, la fonction publique lato sensu, où se croisent fonctionnaires, agents contractuels de droit public (eux-mêmes en contrat à durée déterminée ou indéterminée), ou agents contractuels de droit privé (bénéficiaires de contrats aidés, intérimaires...). À titre d'exemple, l'article L. 1432-9 du Code de la santé publique prévoit expressément que «le personnel de l'agence comprend : $1^{\circ}$ Des fonctionnaires ; (...) $3^{\circ}$ Des agents contractuels de droit public ; $4^{\circ}$ Des agents de droit privé régis par les conventions collectives applicables au personnel des organismes de sécurité sociale.» 
Les deux exemples qui peuvent en être donnés sont ceux des entreprises France Télécom / Orange et La Poste. Dans les deux cas, leur transformation en exploitants publics sous la forme d'établissements publics, puis en sociétés commerciales, a conduit à ce que leur personnel soit composé à la fois d'agents de droit privé et de fonctionnaires continuant à être régis par leurs statuts particuliers ${ }^{24}$. La question s'est alors posée de savoir comment organiser la participation des travailleurs de ces entreprises. Fallait-il leur appliquer les modalités de participation tirées du droit de la fonction publique ? Fallait-il, au contraire, généraliser les modalités de participation prévues par le Code du travail ? Fallait-il organiser la cohabitation de modalités de participation propres à chaque catégorie de personnels?

Dans le cas de La Poste, le choix du législateur a été de maintenir, tout en l'amendant, le modèle tiré du droit de la fonction publique (comités techniques, commissions paritaires, comité d'hygiène, de sécurité et des conditions de travail). Ainsi, le fait pour l'entreprise La Poste de recruter des « agents contractuels sous le régime des conventions collectives (...) n'a pas pour effet de rendre applicables à La Poste les dispositions du Code du travail relatives aux comités d'entreprise, ni celles relatives aux délégués du personnel et aux délégués syndicaux $»^{25}$.

La représentation des agents de droit privé au sein des instances de concertation n'en fait pas moins l'objet d'une attention particulière. Elle passe par une représentation dite " collective » au sein des comités techniques et du comité d'hygiène, de sécurité et des conditions de travail, dont les membres sont élus par l'ensemble des agents, fonctionnaires et agents de droit privé ${ }^{26}$. Elle passe aussi par une représentation dite «individuelle » exigée par la loi de 1990 elle-même, dont l'article 31 prévoit qu'« un décret en Conseil d'État (...) précise en outre, en tenant compte de l'objectif d'harmoniser au sein de La Poste les institutions représentatives du personnel, les conditions dans lesquelles la représentation individuelle des agents de droit privé est assurée, et établit les règles de protection, au moins équivalentes à celles prévues par le Code du travail pour les délégués du personnel, dont bénéficient leurs représentants ». Cette représentation individuelle est organisée par le décret $n^{\circ} 2014-1426$ du 28 novembre 2014 relatif à la représentation des agents contractuels. L'article 2 du décret prévoit que « La représentation individuelle des agents contractuels de La Poste est assurée, au niveau national et aux niveaux opérationnels déconcentrés, au sein de commissions consultatives paritaires » dont l'avis est requis avant toute décision de licenciement, avant le prononcé des sanctions les plus graves ou, à la demande de l'agent, à propos de décisions relatives à sa carrière (évaluation, promotion, formation, etc.).

24 Article 9 de la loi n $90-568$ du 2 juillet 1990 relative à l'organisation du service public de La Poste et à France Télécom. Cette même loi prévoit cependant le rattachement des corps de fonctionnaires à chacune des deux entreprises (article 29-1 pour France Télécom, article 29-4 pour La Poste).

25 Loi préc., article 31.

26 Article 12 du décret $n^{\circ}$ 2011-1063 du 7 septembre 2011 relatif aux comités techniques de La Poste: "Sont électeurs pour la désignation des représentants du personnel au sein du comité technique tous les agents exerçant leurs fonctions dans le périmètre du ou des services au titre desquels le comité est institué.» 
S'agissant de France Télécom / Orange, c'est une autre option qui a été retenue ${ }^{27}$. Après une pério de au cours de laquelle une instance de participation ad hoc a été mise en place ${ }^{28}$, le choix a été fait, en $2003^{29}$, de rendre applicable à l'ensemble du personnel le droit commun de la représentation, sans pour autant renoncer à certaines spécificités institutionnelles ${ }^{30}$. Ainsi, l'article 29-1 alinéa 4 de la loi du 2 juillet 1990 relative à l'organisation du service public de la poste et à France Télécom prévoit que «Par dérogation à l'article 9 de la loi n 83-634 du 13 juillet 1983 précitée et au chapitre II de la loi n 84-16 du 11 janvier 1984 précitée, les fonctionnaires de France Télécom participent avec les salariés de l'entreprise à l'organisation et au fonctionnement de leur entreprise, ainsi qu'à la gestion de son action sociale, par l'intermédiaire des institutions représentatives prévues aux titres ler à IV du livre III de la deuxième partie du Code du travail [délégués du personnel et comité d'entreprise], sous réserve des adaptations, précisées par décret en Conseil d'Etat, qui sont justifiées par la situation particulière des fonctionnaires de France Télécom » (nous soulignons). Alors même que les urnes sont séparées, la Cour de cassation est venue préciser que tous les travailleurs sont réputés former un collège unique, de sorte (c'était l'enjeu) que si les votes des fonctionnaires ne peuvent être pris en compte pour apprécier la représentativité des syndicats au niveau de la branche ou au niveau national, ils le sont, avec ceux des salariés de droit privé, pour apprécier la représentativité des syndicats dans l'entreprise ${ }^{31}$. La Cour rejoint ici la jurisprudence du Conseil constitutionnel en vertu de laquelle le principe de participation n'impose nullement la constitution de collèges distincts dans les instances

27 La loi du 2 juillet 1990 ne qualifie plus France Télécom d'«entreprise nationale » depuis le $1^{\text {er }}$ janvier 2004. Jusque-là, l'article 1-1 de la loi prévoyait que «La personne morale de droit public France Télécom mentionnée à l'article 1er est transformée à compter du 31 décembre 1996 en une entreprise nationale dénommée France Télécom, dont l'Etat détient directement ou indirectement plus de la moitié du capital social. » Les participations cumulées de l'État et de BPI France dépassent seulement les $20 \%$. Pour autant, « la position d'unique actionnaire de référence donne à l'État une influence sur la gouvernance supérieure à ce que son niveau de participation laisserait attendre. Elle lui donne également une possibilité de veto, de fait, sur les décisions qui relèvent de l'assemblée générale et lui permet de s'opposer à une prise de contrôle qu'il considérerait contraire aux intérêts du pays " (Cour des comptes, L'État actionnaire, Rapport public thématique, janv. 2017, p. 197-198).

28 De 1996 à 2004, l'article 29-1 de la loi du 2 juillet 1990 relative à l'organisation du service public de la poste et à France Télécom prévoyait qu'un comité paritaire, informé et consulté notamment sur l'organisation, la gestion et la marche générale de l'entreprise ainsi que sur les questions relatives au recrutement des personnels et les projets de statuts particuliers, comprendraient des représentants de l'entreprise, un collège représentant les agents fonctionnaires et un collège représentant les agents relevant de la convention collective ainsi que les agents non titulaires de droit public. Ces deux collèges se répartissaient les sièges réservés aux représentants des personnels en tenant compte de la proportion de chacune des deux catégories dans l'effectif global de l'entreprise nationale.

29 Loi $n^{\circ}$ 2003-1365 du 31 décembre 2003 relative aux obligations de service public des télécommunications et à France Télécom.

30 Ainsi du conseil des questions statutaires d'Orange SA, qui « connaît des questions et projets de texte relatifs aux règles statutaires applicables aux fonctionnaires de France Télécom » (décret $n^{\circ}$ 2017-394 du 24 mars 2017 relatif au conseil des questions statutaires d'Orange SA, art. 5).

31 Cour cass, 2 juillet 2012, avis n 12-00.006, RFDA, 2012, p. 991, rapport Y. Struillou. Les votes des fonctionnaires sont aussi comptabilisés, naturellement, s'agissant de la représentativité des syndicats dans les instances propres à la fonction publique. 


\section{PARTICIPATION DANS L'ENTREPRISE PUbLIOUE EN FrANCE}

représentatives des personnels des entités regroupant fonctionnaires et agents soumis à des conventions collectives ${ }^{32}$.

Quant à la représentation des personnels au conseil d'administration de l'entreprise, l'article $1^{\text {er }}$ du décret $n^{\circ} 2004-977$ du 17 septembre 2004 précise que « les fonctionnaires de France Télécom sont assimilés à des salariés. Les administrateurs élus en application des articles précités le sont par un corps électoral unique composé des fonctionnaires de France Télécom, des agents contractuels de droit public et des agents contractuels employés sous le régime des conventions collectives. »

Au terme de ce rapide survol, l'on ne peut guère que conclure à l'existence de particularités, plus ou moins fortes, en matière de participation des travailleurs au sein des grandes entreprises du secteur public. La présence de la puissance publique, pour n'être jamais neutre, ne suffit pas à expliquer, encore moins à ordonner des spécificités qui tiennent plus à la structure, à la composition, à la culture ou à l'histoire propres de chacune de ces entreprises qu'à un hypothétique «modèle » de participation.

32 CC 2010-91 QPC, 28 janv. 2011, Fédération nationale CGT des personnels des organismes sociaux. À propos de l'Agence régionale de santé, le Conseil souligne que « le principe de participation à la détermination des conditions de travail n'imposait pas au législateur de prévoir l'existence de collèges électoraux distincts pour la désignation des représentants des personnels » (const. 4).

\section{SYLVAIN NIQUÈGE}

Professeur à I'Université de Bordeaux, Institut Léon Duguit (ILD EA 7439).

Thèmes de recherche : Droit administratif général et droit de la fonction publique.

\section{Publications :}

" L'approfondissement des droits statutaires depuis la loi du 20 avril 2016 relative à la déontologie et aux droits et obligations des fonctionnaires ", Revue française du droit administratif, à paraître, 2018.

$\sim$ (dir.), Les figures de la loyauté en droit public, éditions Mare § Martin, 2017, 514 p. 